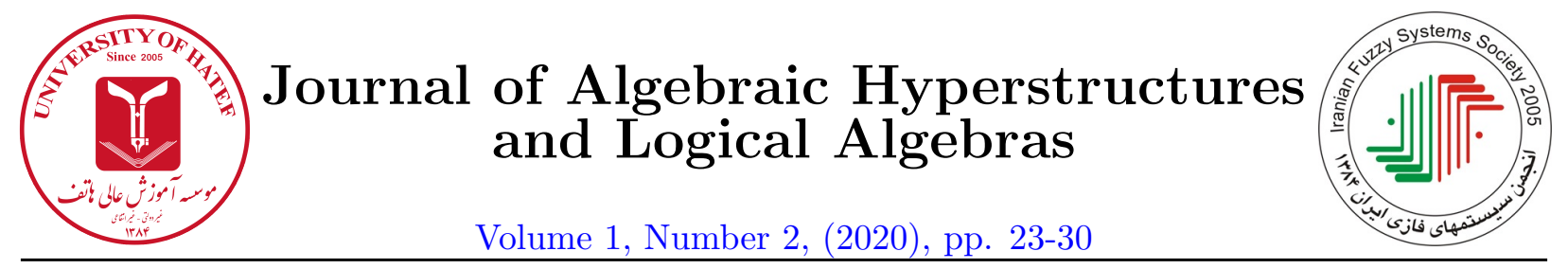

\title{
Some properties of $n$-hyperideals in commutative hyperrings
}

\author{
S. Omidi ${ }^{1}$, B. Davvaz ${ }^{2}$ and J. Zhan ${ }^{3}$ \\ ${ }^{1,2}$ Department of Mathematics, Yazd University, Yazd, Iran \\ ${ }^{3}$ Department of Mathematics, Hubei University for Nationalities, Enshi, Hubei Province 445000, China \\ omidi.saber@yahoo.com,davvaz@yazd.ac.ir, zhanjianming@hotmail.com
}

\begin{abstract}
In this paper, we reformulate several results in commutative algebra in terms of commutative hyperrings. We introduce n-hyperideals in commutative hyperrings and give its some basic properties. Based on new definitions and theorems, we obtain some results in the hyperring theory. Also, the paper is stated a characterization for fundamental n-hyperideals.
\end{abstract}

\section{Article Information}

Corresponding Author:

B. Davvaz;

Received: May 2020;

Accepted: Invited paper.

Paper type: Original.

\section{Keywords:}

Commutative hyperring, homomorphism, n-hyperideal.

\section{Introduction}

The theory of hyperstructures was introduced by Marty [10] at the $8^{\text {th }}$ congress of Scandinavian Mathematicians in 1934. Some review of the hyperstructure theory can be found in [3, 4, 5, 6, 15, Mittas [1] introduced the notion of canonical hypergroups. Hyperrings and hyperfields were introduced by Krasner [9] in connection with his work on valued fields. Davvaz and Leoreanu studied hyperrings in more details in [6]. Several kinds of hyperrings are introduced and analyzed. Ameri and Norouzi [1] studied homomorphisms of hyperring and extension (contraction) of hyperideals in commutative hyperrings. In 2015, Jun [8] studied algebraic and geometric aspects of hyperrings. He introduced the notion of an integral hyperring scheme $\left(X, \mathcal{O}_{X}\right)$ and proved that $\Gamma\left(X, \mathcal{O}_{X}\right) \simeq R$ for any integral affine hyperring scheme $X=\operatorname{Spec}(R)$. In [12], some results concerning ordered hyperstructures are proved. Some results on a derivation in hyperrings can be found in [2]. Recently, Tekir et al. [13] introduced the concept of n-ideals on commutative rings.

Let $R$ be a commutative Krasner hyperring with nonzero identity. In this paper, we generalize some concepts of the ring theory such as n-ideals and r-ideals on hyperrings. Also, we investigate some properties of n-hyperideals analogous with prime hyperideals in commutative hyperrings.

https://doi.org/10.29252/HATEF.JAHLA.1.2.3 


\section{Preliminaries}

Let $H$ be a non-empty set and $\mathcal{P}^{*}(H)$ denotes the family of all non-empty subsets of $H$. A mapping $\circ: H \times H \rightarrow \mathcal{P}^{*}(H)$ is called a binary hyperoperation on $H$. The couple $(H, \circ)$ is called a hypergroupoid. In the above definition, if $A$ and $B$ are two non-empty subsets of $H$ and $x \in H$, then we define:

$$
A \circ B=\bigcup_{\substack{a \in A \\ b \in B}} a \circ b, A \circ x=A \circ\{x\} \text { and } x \circ B=\{x\} \circ B \text {. }
$$

A hypergroupoid $(H, \circ)$ is said to be a semihypergroup if for all $x, y, z \in H,(x \circ y) \circ z=x \circ(y \circ z)$, which means that

$$
\bigcup_{u \in x \circ y} u \circ z=\bigcup_{v \in y \circ z} x \circ v .
$$

A non-empty subset $K$ of a semihypergroup $(H, \circ)$ is called a subsemihypergroup of $H$ if $K \circ K \subseteq K$. A semihypergroup $(H, \circ)$ satisfying $x \circ H=H \circ x=H$ for any $x \in H$ is called a hypergroup. A non-empty subset $K$ of $H$ is a subhypergroup of $H$ if $a \circ K=K \circ a=K$, for all $a \in K$.

Now, we introduce the notions of canonical hypergroups and Krasner hyperrings and we apply them in the next section.

Definition 2.1. [11] A non-empty set $R$ along with the hyperoperation + is called a canonical hypergroup if the following axioms hold:

(1) $x+(y+z)=(x+y)+z$, for any $x, y, z \in R$;

(2) $x+y=y+x$, for any $x, y \in R$;

(3) there exists $0 \in R$ such that $x+0=\{x\}$, for any $x \in R$;

(4) for any $x \in R$, there exists a unique element $x^{\prime} \in R$, such that $0 \in x+x^{\prime}$ (we shall write $-x$ for $x^{\prime}$ and we call it the opposite of $x$ );

(5) $z \in x+y$ implies that $y \in-x+z$ and $x \in z-y$, that is $(R,+)$ is reversible.

Definition 2.2. 9] A Krasner hyperring is an algebraic hypersructure $(R,+, \cdot)$ which satisfies the following axioms:

(1) $(R,+)$ is a canonical hypergroup;

(2) $(R, \cdot)$ is a semigroup having 0 as a bilaterally absorbing element, i.e., $x \cdot 0=0 \cdot x=0$, for all $x \in R$;

(3) $(y+z) \cdot x=(y \cdot x)+(z \cdot x)$ and $x \cdot(y+z)=(x \cdot y)+(x \cdot z)$, for all $x, y, z \in R$.

A Krasner hyperring $R$ is called with identity if there exists an element, say $1 \in R$, such that $1 \cdot x=x \cdot 1=x$. An element $x$ of a Krasner hyperring $R$ is called a unit if there exists $y \in R$ such that $x \cdot y=y \cdot x=1$. A Krasner hyperring $R$ is called commutative (with unit element) if $(R, \cdot)$ is a commutative semigroup (with unit element). A Krasner hyperring $R$ is called a Krasner hyperfield, if $(R \backslash\{0\}, \cdot)$ is a group. A Krasner hyperring $R$ is called a hyperdomain, if $R$ is a commutative hyperring with unit element and $a \cdot b=0$ implies that $a=0$ or $b=0$, for all $a, b \in R$. A subhyperring of a Krasner hyperring $(R,+, \cdot)$ is a non-empty subset $A$ of $R$ which 
forms a Krasner hyperring containing 0 under the hyperoperation + and the operation $\cdot$ on $R$, that is, $A$ is a canonical subhypergroup of $(R,+)$ and $A \cdot A \subseteq A$. Then a non-empty subset $A$ of $R$ is a subhyperring of $(R,+, \cdot)$ if and only if, for all $x, y \in A, x+y \subseteq A,-x \in A$ and $x \cdot y \in A$. A non-empty subset $I$ of $(R,+, \cdot)$ is called a left (resp. right) hyperideal of $(R,+, \cdot)$ if $(I,+)$ is a canonical subhypergroup of $(R,+)$ and for any $a \in I$ and $r \in R, r \cdot a \in I$ (resp. $a \cdot r \in I$ ). A hyperideal $I$ of $(R,+, \cdot)$ is one which is a left as well as a right hyperideal of $R$, that is, $x+y \subseteq I$ and $-x \in I$, for all $x, y \in I$ and $x \cdot y, y \cdot x \in I$, for all $x \in I$ and $y \in R$. Throughout this paper, unless otherwise stated, $R$ is always a commutative Krasner hyperring with nonzero identity.

Lemma 2.3. 6] A non-empty subset $A$ of a Krasner hyperring $R$ is a left (resp. right) hyperideal if and only if

(1) $a, b \in A$ implies $a-b \subseteq A$.

(2) $a \in A$ and $r \in R$ imply $r \cdot a \in A$ (resp. $a \cdot r \in A)$.

Definition 2.4. A homomorphism from a Krasner hyperring $(R,+, \cdot)$ into a Krasner hyperring $(S, \oplus, \odot)$ is a mapping $\varphi: R \rightarrow S$ such that we have:

(1) $\varphi(a+b) \subseteq \varphi(a) \oplus \varphi(b)$;

(2) $\varphi(a \cdot b)=\varphi(a) \odot \varphi(b)$.

Also, $\varphi$ is called a good homomorphism if in the previous condition (1), the equality is valid.

\section{3 n-Hyperideals of commutative hyperrings}

Recall that a proper hyperideal $\mathfrak{p}$ of a commutative hyperring $(R,+, \cdot)$ is called prime if $a \cdot b \in \mathfrak{p}$ implies that either $a \in \mathfrak{p}$ or $b \in \mathfrak{p}$. Let $R$ be a commutative hyperring with identity. By $\operatorname{Spec}(R)$ we mean the set of all the prime hyperideals of $R$. For hyperideal $I$ of $R$ we define $V(I)$ as follows:

$$
V(I):=\{\mathfrak{p} \in \operatorname{Spec}(R) \mid I \subseteq \mathfrak{p}\} .
$$

For $a \in R$, we set $V(a):=\{\mathfrak{p} \in \operatorname{Spec}(R) \mid a \in \mathfrak{p}\}$. Then, $V(I)=\bigcap_{a \in I} V(a)$.

Lemma 3.1. 8] Let $I$ be a hyperideal of a hyperring $R$. Then

$$
\sqrt{I}:=\left\{r \in R \mid \exists n \in \mathbb{N} \text { such that } r^{n} \in I\right\} .
$$

is a hyperideal.

Lemma 3.2. [8] Let I be a hyperideal of a hyperring $R$. Then

$$
\sqrt{I}=\bigcap_{\mathfrak{p} \in V(I)} \mathfrak{p} .
$$

Definition 3.3. A hyperideal $I$ of a Krasner hyperring $(R,+, \cdot)$, such that $I \neq R$, is called an $n$-hyperideal if for $a, b$ of $R, a \cdot b \in I$ and $a \notin \sqrt{0}$ implies that $b \in I$.

Example 3.4. Let $R=\{0, a, b\}$ be a set with the hyperaddition + and the multiplication $\cdot$ defined as follows:

\begin{tabular}{|c|ccc|}
\hline+ & 0 & $a$ & $b$ \\
\hline 0 & 0 & $a$ & $b$ \\
$a$ & $a$ & $R$ & $a$ \\
$b$ & $b$ & $a$ & $\{0, b\}$ \\
\hline
\end{tabular}

\begin{tabular}{|c|ccc|}
\hline$\cdot$ & $a$ & $b$ & $c$ \\
\hline 0 & 0 & 0 & 0 \\
$a$ & 0 & $a$ & $b$ \\
$b$ & 0 & $b$ & 0 \\
\hline
\end{tabular}

Then, $(R,+, \cdot)$ is a Krasner hyperring. It is easy to see that $\{0\}$ and $\{0, b\}$ are n-hyperideals of $R$. 
Lemma 3.5. Let $(R,+, \cdot)$ be a hyperring. Then,

(1) If $\left\{I_{k} \mid k \in \Omega\right\}$ is a family of n-hyperideals of $R$ such that $I_{i} \subseteq I_{j}$ or $I_{j} \subseteq I_{i}$ for all $i, j \in \Omega$, then $\bigcup_{k \in \Omega} I_{k}$ is an n-hyperideal of $R$.

(2) If $\left\{I_{k} \mid k \in \Omega\right\}$ is a family of $n$-hyperideals of $R$, then $\bigcap_{k \in \Omega} I_{k}$ is an n-hyperideal of $R$.

Proof. (1): Since $0 \in \bigcup_{k \in \Omega} I_{k}$, it follows that $\bigcup_{k \in \Omega} I_{k} \neq \emptyset$. Let $x, y \in \bigcup_{k \in \Omega} I_{k}$. Then $x, y \in I_{k}$ for some $k \in \Omega$. Since $I_{k}$ is a hyperideal of $R$, we obtain $x-y \subseteq I_{k}$ for some $k \in \Omega$. Thus $x-y \subseteq \bigcup_{k \in \Omega} I_{k}$. Also, $\left(\bigcup_{k \in \Omega} I_{k}\right) \cdot R=\bigcup_{k \in \Omega} I_{k} \cdot R \subseteq \bigcup_{k \in \Omega} I_{k}$ and $R \cdot\left(\bigcup_{k \in \Omega} I_{k}\right)=\bigcup_{k \in \Omega} R \cdot I_{k} \subseteq \bigcup_{k \in \Omega} I_{k}$. So, for each $x \in \bigcup_{k \in \Omega} I_{k}$ and $s \in R, x \cdot s \in \bigcup_{k \in \Omega} I_{k}$. Similarly, $s \cdot x \in \bigcup_{k \in \Omega} I_{k}$. Now, let $a \cdot b \in \bigcup_{k \in \Omega} I_{k}$ and $a \notin \sqrt{0}$ for $a, b \in R$. Then, $a \cdot b \in I_{i}$ for some $i \in \Omega$. Since $I_{i}$ is an n-hyperideal of $R$, it follows that $b \in I_{i} \subseteq \bigcup_{k \in \Omega} I_{k}$. Therefore, $\bigcup_{k \in \Omega} I_{k}$ is an n-hyperideal of $R$.

(2): The proof is straightforward.

The set $\operatorname{ann}(x)=\{a \in R \mid a \cdot x=0\}$ is called the annihilator of $x$ in $R$. A proper hyperideal $I$ of a hyperring $(R,+, \cdot)$ is said to be an $r$-hyperideal of $R$ if $x \cdot y \in I$ and ann $(x)=0$ imply that $y \in I$ for any $x, y \in R$. Every n-hyperideal of a hyperring $R$ is an r-hyperideal of $R$. The converse is not true, in general, that is, an r-hyperideal may not be an n-hyperideal of $R$. The following example denotes such a situation.

Example 3.6. Let $R=\{0, a, b, c\}$ be a set with the hyperaddition + and the multiplication $\cdot$ defined as follows:

\begin{tabular}{|c|cccc|}
\hline+ & 0 & $a$ & $b$ & $c$ \\
\hline 0 & 0 & $a$ & $b$ & $c$ \\
$a$ & $a$ & $\{0, b\}$ & $\{a, c\}$ & $b$ \\
$b$ & $b$ & $\{a, c\}$ & $\{0, b\}$ & $a$ \\
$c$ & $c$ & $b$ & $a$ & 0 \\
\hline
\end{tabular}

\begin{tabular}{|l|llll|}
\hline$\cdot$ & 0 & $a$ & $b$ & $c$ \\
\hline 0 & 0 & 0 & 0 & 0 \\
$a$ & 0 & $a$ & $b$ & $c$ \\
$b$ & 0 & $b$ & $b$ & 0 \\
$c$ & 0 & $c$ & 0 & $c$ \\
\hline
\end{tabular}

Then, $(R,+, \cdot)$ is a Krasner hyperring [2]. Clearly, $\{0\},\{0, b\}$ and $\{0, c\}$ are proper hyperideals of $R$. It is easy to see that $\{0, b\}$ is an r-hyperideal of $R$, but it is not an n-hyperideal of $R$. Indeed:

$$
b \cdot c=0 \in\{0, b\} \text { and } b \notin \sqrt{0_{R}} \text { but } c \notin\{0, b\} .
$$

Theorem 3.7. Let $\mathfrak{p}$ be a prime hyperideal of a hyperring $(R,+, \cdot)$. Then $\mathfrak{p}$ is an n-hyperideal of $R$ if and only if $\mathfrak{p}=\sqrt{0}$.

Proof. By Lemma $3.2, \sqrt{0}=\bigcap_{\mathfrak{p} \in \operatorname{Spec}(R)} \mathfrak{p} \subseteq \mathfrak{p}$. Let $\mathfrak{p} \nsubseteq \sqrt{0}$. Then there exists $a \in \mathfrak{p}$ such that $a \notin \sqrt{0}$. Since $\mathfrak{p}$ is an n-hyperideal of $R$ and $a \cdot 1=a \in \mathfrak{p}$, we get $1 \in \mathfrak{p}$. Thus, $I=R$, a contradiction. Hence, $\mathfrak{p} \subseteq \sqrt{0}$ which implies that $\mathfrak{p}=\sqrt{0}$.

Conversely, let $a \cdot b \in \mathfrak{p}$ and $a \notin \sqrt{0}=\mathfrak{p}$ for $a, b \in R$. Since $\mathfrak{p}$ is a prime hyperideal of $R$, we have $b \in \mathfrak{p}$. Therefore, $\mathfrak{p}$ is a prime hyperideal of $R$.

Example 3.8. In Example 3.6, $\{0, b\}$ is a prime hyperideal of $R$, but it is not an n-hyperideal of $R$. 
For a (multiplicative) submonoid $S$ of a hyperring $R$, let us consider the following relation in $R \times S:$

$$
(r, s) \sim\left(r^{\prime}, s^{\prime}\right) \Leftrightarrow \exists x \in S \text { s.t. } x r s^{\prime}=x r^{\prime} s .
$$

Clearly, $\sim$ is an equivalence relation on $R \times S$. Let $[(r, s)]$ be the equivalence relation of $(r, s) \in$ $R \times S . S^{-1} R$ is the set $(R \times S / \sim)$. Now, we define the following hyperoperation $\oplus$ and operation $\odot$ on $S^{-1} R$,

$$
[(r, s)] \oplus\left[\left(r^{\prime}, s^{\prime}\right)\right]=\left\{\left[\left(y, s \cdot s^{\prime}\right)\right] \mid y \in r \cdot s^{\prime}+r^{\prime} \cdot s\right\}
$$

and

$$
[(r, s)] \odot\left[\left(r^{\prime}, s^{\prime}\right)\right]=\left\{\left[\left(r \cdot r^{\prime}, s \cdot s^{\prime}\right)\right]\right\}
$$

Clearly, $\left(S^{-1} R, \oplus, \odot\right)$ is a commutative hyperring [7]. The mapping $\varphi: R \rightarrow S^{-1} R$ given by $\varphi(r)=r / 1$ is a homomorphism. If $I$ is a hyperideal of $R$, then

$$
\varphi(I)=S^{-1} I=\left\{\lambda \in S^{-1} R \mid \lambda=a / s, \exists a \in I, \exists s \in S\right\}
$$

is a hyperideal of $S^{-1} R . S^{-1} I$ is called the extension of $I$ in $S^{-1} R$.

Theorem 3.9. If $I$ is an n-hyperideal of a hyperring $(R,+, \cdot)$, then $S^{-1} I$ is an n-hyperideal of $S^{-1} R$.

Proof. Let $r / s \odot r^{\prime} / s^{\prime} \in S^{-1} I$ and $r / s \notin \sqrt{0_{S^{-1} R}}$ for $r, r^{\prime} \in R$ and $s, s^{\prime} \in S$. Then there exists $u \in S$ such that $u r r^{\prime} \in I$. Next, we show that $r \notin \sqrt{0_{R}}$. If $r \in \sqrt{0_{R}}$, then there exists $n \in \mathbb{N}$ such that $r^{n}=0_{R}$. This means that $(r / 1)^{n}=r^{n} / 1=0_{R} / 1=0_{S^{-1} R}=0_{R} / s$, and so $r / 1 \in \sqrt{0_{S^{-1} R}}$. Since $r / s=1 / s \odot r / 1$, we get $(r / s)^{n}=(1 / s)^{n} \odot 0_{S^{-1} R}=0_{S^{-1} R}$. Hence, $r / s \in \sqrt{0_{S^{-1} R}}$, which is a contradiction. This implies that $r \notin \sqrt{0_{R}}$. Now, since $I$ is an n-hyperideal of $R$, we have $u r^{\prime} \in I$ and so $r^{\prime} / s^{\prime}=u r^{\prime} / u s^{\prime} \in S^{-1} I$. Therefore, $S^{-1} I$ is an n-hyperideal of $S^{-1} R$.

Theorem 3.10. Let $I$ be an n-hyperideal of the hyperring $(R,+, \cdot)$ and $\varphi: R \rightarrow S$ a good epimorphism such that $\operatorname{Ker} \varphi \subseteq I$. Then $\varphi(I)$ is an n-hyperideal of the hyperring $(S, \oplus, \odot)$.

Proof. Clearly, $\varphi(I)$ is a hyperideal of $S$. Let $s_{1} \odot s_{2} \in \varphi(I)$ and $s_{1} \notin \sqrt{0_{S}}$ for $s_{1}, s_{2} \in S$. Then, there exist $r_{1}, r_{2} \in R$ such that $s_{1}=\varphi\left(r_{1}\right)$ and $s_{2}=\varphi\left(r_{2}\right)$ (since $\varphi$ is onto) which

$$
s_{1} \odot s_{2}=\varphi\left(r_{1}\right) \odot \varphi\left(r_{2}\right)=\varphi\left(r_{1} \cdot r_{2}\right)=\varphi(x) \in \varphi(I)
$$

for some $x \in I$. So, we have

$$
0 \in \varphi\left(r_{1} \cdot r_{2}\right) \ominus \varphi(x)=\varphi\left(r_{1} \cdot r_{2}-x\right) .
$$

Hence, there exists $t \in r_{1} \cdot r_{2}-x$ such that $\varphi(t)=0$. By hypothesis, we have

$$
r_{1} \cdot r_{2} \in t+x \subseteq \operatorname{Ker} \varphi+I \subseteq I+I \subseteq I .
$$

So, $r_{1} \cdot r_{2} \in I$. Next, we show that $r_{1} \notin \sqrt{0_{R}}$. If $r_{1} \in \sqrt{0_{R}}$, then there exists $n \in \mathbb{N}$ such that $r_{1}^{n}=0_{R}$. This means that $\varphi\left(r_{1}^{n}\right)=\varphi(0)=0_{S}$, and so $\left(\varphi\left(r_{1}\right)\right)^{n}=0_{S}$. Hence, $s_{1}=\varphi\left(r_{1}\right) \in \sqrt{0_{S}}$, which is a contradiction. This implies that $r_{1} \notin \sqrt{0_{R}}$. Now, since $I$ is an n-hyperideal of $R$, we get $r_{2} \in I$ and so $s_{2}=\varphi\left(r_{2}\right) \in \varphi(I)$. This completes the proof.

Let $\varphi: R \rightarrow S$ be a homomorrphism of hyperrings and $I$ a hyperideal of $R$. The hyperideal $\langle\varphi(I)\rangle$ of $S$ generated by the set $\varphi(I)$ is called the extension of $I$, and is denoted by $I^{e}$. We have 


$$
\langle\varphi(I)\rangle=\left\{x \in S \mid x \in \sum_{i=1}^{n} s_{i} \cdot \varphi\left(a_{i}\right), s_{i} \in S, a_{i} \in I, n \in \mathbb{N}\right\} .
$$

The mapping $\varphi: R \rightarrow S^{-1} R$ given by $\varphi(r)=r / 1$ is a homomorphism. Consider $\lambda \in S^{-1} I$. Then $\lambda=i / s$, where $i \in I$ and $s \in S$. Hence, $i / 1 \in \varphi(I)$. This implies that $i / 1 \in I^{e}$. Since $I^{e}$ is a hyperideal of $S^{-1} R$, we get $i / s=1 / s \odot i / 1 \in I^{e}$. So, $\lambda=i / s \in I^{e}$. Thus, $S^{-1} I \subseteq I^{e}$. Now, suppose that $\lambda \in \varphi(I)$. Then there exists $a \in I$ such that $\lambda=a / 1$. Hence, $\lambda \in S^{-1} I$ which implies that $\varphi(I) \subseteq S^{-1} I$. Thus, $I^{e}=\langle\varphi(I)\rangle \subseteq S^{-1} I$. Hence, $S^{-1} I=I^{e}$.

Theorem 3.11. Let $I$ be an n-hyperideal of the hyperring $(R,+, \cdot)$ and $\varphi: R \rightarrow S$ a good epimorphism such that Ker $\varphi \subseteq I$. Then $I^{e}$ is an n-hyperideal of the hyperring $(S, \oplus, \odot)$.

Proof. The proof is similar to the proof of Theorem 3.10 .

Theorem 3.12. Let $J$ be an n-hyperideal of the hyperring $(S, \oplus, \odot)$ and $\varphi: R \rightarrow S$ a good monomorphism. Then $\varphi^{-1}(J)=\{a \in R \mid \varphi(a) \in J\}$ is an n-hyperideal of the hyperring $(R,+, \cdot)$. $\varphi^{-1}(J)$ is called the contraction of $J$, and is denoted by $J^{c}$.

Proof. Since $0 \in \varphi^{-1}(J)$, it follows that $\varphi^{-1}(J) \neq \emptyset$. Let $x \in R$. Since $\varphi$ is a homomorphism and $0 \in x-x$, we have $0=\varphi(0) \in \varphi(x-x) \subseteq \varphi(x) \oplus \varphi(-x)$. So $0 \in \varphi(x) \oplus \varphi(-x)$. Thus, $\varphi(-x)$ is the inverse of $\varphi(x)$ in the canonical hypergroup $(S, \oplus)$. Since $0 \in \varphi(x) \oplus \varphi(-x)$, it follows that $\varphi(-x)=-\varphi(x)$. Now, let $a_{1}, a_{2} \in \varphi^{-1}(J)$. Then $\varphi\left(a_{1}\right), \varphi\left(a_{2}\right) \in J$. Since $J$ is a hyperideal of $T$, we have $\varphi\left(a_{1}-a_{2}\right) \subseteq \varphi\left(a_{1}\right) \ominus \varphi\left(a_{2}\right) \subseteq J$. Hence $a_{1}-a_{2} \subseteq \varphi^{-1}(J)$. Let $x \in R$ and $a \in \varphi^{-1}(J)$. Then $\varphi(a) \in J$. Since $\varphi$ is a homomorphism, it follows that $\varphi(x \cdot a)=\varphi(x) \odot \varphi(a) \in J$. Thus $x \cdot a \in \varphi^{-1}(J)$. Hence, $\varphi^{-1}(J)$ is a hyperideal of $R$. Now, let $a \cdot b \in \varphi^{-1}(J)$ and $a \notin \sqrt{0_{R}}$. Then $\varphi(a) \odot \varphi(b)=\varphi(a \cdot b) \in J$. Next, we show that $\varphi(a) \notin \sqrt{0_{S}}$. If $\varphi(a) \in \sqrt{0_{S}}$, then there exists $n \in \mathbb{N}$ such that $(\varphi(a))^{n}=0_{S}$. This means that $\varphi\left(a^{n}\right)=0_{S}=\varphi\left(0_{R}\right)$, and so $a^{n}=0_{R}$. Hence, $a \in \sqrt{0_{R}}$, which is a contradiction. This leads to $\varphi(a) \notin \sqrt{0_{S}}$. Now, since $J$ is an n-hyperideal of $S$, we get $\varphi(b) \in J$ and so $b \in \varphi^{-1}(J)$. Therefore, $\varphi^{-1}(J)$ is an n-hyperideal of $R$.

A relation $\sigma^{*}$ is the transitive closure of a binary relation $\sigma$ if (1) $\sigma^{*}$ is transitive; (2) $\sigma \subseteq \sigma^{*}$ and (3) for any relation $\sigma^{\prime}$, if $\sigma \subseteq \sigma^{\prime}$ and $\sigma^{\prime}$ is transitive, then $\sigma^{*} \subseteq \sigma^{\prime}$, that is, $\sigma^{*}$ is the smallest relation that satisfies $(1)$ and $(2)$. Let $(R,+, \cdot)$ be a hyperring. We define the relation $\gamma$ as follows:

$$
x \gamma y \Leftrightarrow \exists n \in \mathbb{N}, \exists k_{i} \in \mathbb{N}, \exists\left(x_{i 1}, \cdots, x_{i k_{i}}\right) \in R^{k_{i}}, 1 \leq i \leq n,
$$

such that

$$
\{x, y\} \subseteq \sum_{i=1}^{n}\left(\prod_{j=1}^{k_{i}} x_{i j}\right) .
$$

Theorem 3.13. [14] Let $R$ be a hyperring and $\gamma^{*}$ be the transitive closure of $\gamma$. Then, we have:

(1) $\gamma^{*}$ is a strongly regular relation both on $(R,+)$ and $(R, \cdot)$.

(2) The quotient $R / \gamma^{*}$ is a ring.

(3) The relation $\gamma^{*}$ is the smallest equivalence relation such that the quotient $R / \gamma^{*}$ is a ring.

Clearly, $\varphi: R \rightarrow R / \gamma^{*}$ defined by $\varphi(x)=\gamma^{*}(x)$ for all $x \in R$, is a homomorphism. The kernel of $\varphi, \operatorname{ker} \varphi$, is defined by $\operatorname{ker} \varphi=\left\{x \in R \mid \gamma^{*}(x)=\gamma^{*}(0)\right\}$. We denote by $0_{R / \gamma^{*}}$ the zero element of $R / \gamma^{*}$. If $R$ is a Krasner hyperring, then $\gamma^{*}(0)=0_{R / \gamma^{*}}$ and $\gamma^{*}(-x)=-\gamma^{*}(x)$ for all $x \in R$. 
Theorem 3.14. Let $(R,+, \cdot)$ be a Krasner hyperring and $\gamma^{*}$ a fundamental relation on $R$. If I is an n-hyperideal of $R$ such that $\operatorname{Ker} \varphi \subseteq I$, then $\gamma^{*}(I)=\left\{\gamma^{*}(a) \mid a \in I\right\}$ is an n-hyperideal of $R / \gamma^{*}$.

Proof. Clearly, $\gamma^{*}(I)$ is a hyperideal of $R / \gamma^{*}$. Let $\gamma^{*}(a) \odot \gamma^{*}(b) \in \gamma^{*}(I)$ and $\gamma^{*}(a) \notin \sqrt{0_{R / \gamma^{*}}}$ for $\gamma^{*}(a), \gamma^{*}(b) \in R / \gamma^{*}$. Then, there exists $x \in I$ such that $\gamma^{*}(a \cdot b)=\gamma^{*}(a) \odot \gamma^{*}(b)=\gamma^{*}(x)$. So, we have

$$
\gamma^{*}(0)=\gamma^{*}(a \cdot b) \ominus \gamma^{*}(x)=\varphi(a \cdot b) \ominus \varphi(x)=\varphi(a \cdot b-x)=\gamma^{*}(a \cdot b-x) .
$$

Hence, $a \cdot b-x \subseteq \operatorname{Ker} \varphi \subseteq I$. Since $(R,+)$ is a canonical hypergroup, we have

$$
a \cdot b \in a \cdot b+0 \subseteq a \cdot b+x-x \subseteq I+x \subseteq I .
$$

So, $a \cdot b \in I$. Next, we show that $a \notin \sqrt{0_{R}}$. By hypothesis, we have

$$
\gamma^{*}(a) \notin \sqrt{0_{R / \gamma^{*}}}=\sqrt{\gamma^{*}(0)} .
$$

If $a \in \sqrt{0_{R}}$, then there exists $n \in \mathbb{N}$ such that $a^{n}=0$. This means that $\gamma^{*}\left(a^{n}\right)=\gamma^{*}(0)$, and so $\left(\gamma^{*}(a)\right)^{n}=0_{R / \gamma^{*}}$. Hence, $\gamma^{*}(a) \in \sqrt{0_{R / \gamma^{*}}}$, which is a contradiction. This leads to $a \notin \sqrt{0_{R}}$. Now, since $I$ is an n-hyperideal of $R$, we get $b \in I$ and so $\gamma^{*}(b) \in \gamma^{*}(I)$. Therefore, $\gamma^{*}(I)$ is an n-hyperideal of $R / \gamma^{*}$.

\section{Conclusions}

In this paper, we introduced and studied some properties of $n$-hyperideals of commutative hyperrings. Also, we proved that some results on extension (contraction) of $n$-hyperideals in commutative hyperrings. Moreover, we described the behavior of $n$-hyperideals under fundamental relations. We hope that this paper would offer foundation for further study of the theory on commutative hyperrings.

\section{References}

[1] R. Ameri, M. Norouzi, On commutative hyperrings, International Journal of Algebraic Hyperstructures and Its Applications, 1(1) (2014), 45-58.

[2] A. Asokkumar, Derivations in hyperrings and prime hyperrings, Iranian Journal of Mathematical Sciences and Informatics, 8(1) (2013), 1-13.

[3] P. Corsini, Prolegomena of hypergroup theory, Second Edition, Aviani editor, Italy, 1993.

[4] P. Corsini, V. Leoreanu, Applications of hyperstructure theory, Advances in Mathematics, Kluwer Academic Publishers, Dordrecht, 2003.

[5] B. Davvaz, Semihypergroup theory, Elsevier, 2016.

[6] B. Davvaz, V. Leoreanu Fotea, Hyperring theory and applications, International Academic Press, Palm Harbor, USA, 2007.

[7] B. Davvaz, A. Salasi, A realization of hyperrings, Communications in Algebra, 34(12) (2006), 4389-4400.

[8] J. Jun, Algebraic geometry over hyperrings, arXiv preprint arXiv:1512.04837, 2015. 
[9] M. Krasner, A class of hyperrings and hyperfields, International Journal of Mathematics and Mathematical Sciences, 6(2) (1983), 307-311.

[10] F. Marty, Sur une generalization de la notion de groupe, $8^{\text {iem }}$ Congress Math. Scandinaves, Stockholm, 1934, 45-49.

[11] J. Mittas, Hypergroupes canoniques, Mathematica Balkanica, 2 (1972), 165-179.

[12] S. Omidi, B. Davvaz, J. M. Zhan, An investigation on ordered algebraic hyperstructures, Acta Mathematica Sinica, English Series, 2017.

[13] U. Tekir, S. Koc, K. H. Oral, n-ideals of commutative rings, Filomat, 31(10) (2017), 29332941.

[14] T. Vougiouklis, The fundamental relation in hyperrings, The general hyperfields, in: Proc. Fourth Int. Congress on Algebraic Hyperstructures and Applications (AHA 1990), World Scientific, 1991, 203-211.

[15] T. Vougiouklis, Hyperstructures and their representations, Hadronic Press Inc., Florida, 1994. 